\title{
Brain Tumor Segmentation Using Geodesic Region-based Level Set without Re-initialization
}

\author{
Hongzhe Yang ${ }^{1}$, Lihui Zhao ${ }^{2}$ and Songyuan Tang ${ }^{3}$ \\ ${ }^{1}$ School of Computer Science, Beijing Institute of Technology, Beijing, China \\ ${ }^{2}$ School of Electrical Engineering, Liaoning University of Technology, \\ Jinzhou, China \\ ${ }^{3}$ School of Optoelectronics, Beijing Institute of Technology, Beijing, China,
}

\begin{abstract}
Region-based level set segmentation is a paradigm for the automatic segmentation of brain tumor image. Unfortunately, region-based segmentation, which is relied on the intensity difference of different regions, has been of limited used in presence of complex background. In fact, the evoluting curve may leak out the boundary of tumor to reach a steady state by the global region force. In this work, we propose a new hybrid approach for brain tumor segmentation, which is relied on the approach of global intensity difference, local edge properties, curve evolution, and level set method. The regional information drives the contour to converge to the global minimum. By combining the edge information into the region-based framework, the images with intensity inhomogeneity and complex background can be efficiently segmented. To improve the accuracy of brain tumor segmentation, a skull-stripped method for brain images is proposed by utilizing the new morphological process. In addition, a penalizing energy is used for avoiding the time-consuming re-initialization step of the level set method. Finally, experiments are preformatted on some synthetic and real images. By visually assessments, results on patients demonstrate the new method can segment tumors with few iteration times. Moreover, comparisons with the most similar methods also show that the proposed method is effective for the segmentation of tumor in MR image.
\end{abstract}

Keywords: Region-based level set, Geodesic active contour model, Brain tumors, Segmentation, MR image

\section{Introduction}

Region-based level set model is widely utilized method for image segmentation. It relies on the evolution of contours and surfaces which is embedded as the zero level set of a higher dimensional function. To segment a new image, a speed function driven the contour to the object boundary by a time-dependent partial differential equation. Instead of using the image gradient, the region-based level set method use the global information to stabilize the local various of the image (such as weak edge and noise). Among the region-based models, Chan-Vese model [1] which is based on the Mumford-Shah function is a represent method.

Unfortunately, Chan-Vese model is seldom directly used for brain tumor segmentation for some intrinsic limitations. Brain tumor MR image is usually viewed as the complex object image which consists of the white matter, gray matter, cerebrospinal fluid, and abnormal tissues. However, CV is difficult to achieve satisfactory results for the images 
with complex background. This is due to the region energy term drives the contour to the minimum of the intensity difference. In fact, the boundary of desired object is always not the balance points for the intensity difference of the global domain. Moreover, CV model is not suitable for heterogeneous intensity since it assumes that each region always maintain constant intensity. Finally, during the process of the numerical solution of the level set method, periodically re-initializing the level set function to reconstruct the signed distance function is a time-consuming process.

To solve the limitations of CV model, we proposed a new approach which uses both global intensity constrain and local gradient properties for brain tumor segmentation. Local information is adopted in the speed function so that image with heterogeneous intensity can be efficiently segmented. By using the penalizing term, the level set method can be kept stable without the periodically re-initializing. Thus, the algorithm efficiently speeds up the convergence of curve evolution. Consider the synthetic image in Figure 1. The background and foreground are heterogeneous and share nearly the same statistical model. The CV model causes the improperly result and the proposed algorithm achieves good result. Assumed we only want to segment the circle in Figure 2 (b) shows an incorrect segmentation in the image with complex structures on the back ground. The proposed method can efficiently segment star-shape object by combined the edge information with the region information.

The remaining of the paper is organized as follows: In Section 2, we briefly review the state-of-the-art technology. In Section 3 describes the data set used in this work. The proposed model is presented in Section 4. Finally, discussion and conclusion are included in Sections 5 and 6.

\section{Survey of Level Set Methods for Brain Tumor Segmentation}

The contour deformable model is a popular automatic segmentation method. Droske et al., [2] presented glioma segmentation method using the edge-based level set met model. In this method, the contour evolution was determined by the boundary properties in the MRI image. The evolution speed function was a descent function which would take very small values close to the image boundary. Ho et al., [3] proposed a level set method with region competition for automatic brain tumor segmentation. Two T1-weighted image with and without contrast agent are registered for getting a tumor probability map. The probabilities for background and tumor regions are calculated via mixture-modeling of the histogram. Then, the probabilities are used to the region competition snake to segment the tumor structures. Instead of using multichannel image to obtain the poster probabilities, Prastawa et al., [4] presented a brain tumor segmentation framework based on outerlies detection. They first detect tumor and edema region as intensity abnormalities or outerliers, and, then, region competition snake is introduced to provide geometric and spatial constraints for determining proper sample locations. On the other hand, Wang et al., [5] relied on the fluid vector flow active contour model to driven the evolution contour to capture a large range and extract concave shapes.

Cobzas et al., [6] utilized the region-based variational strategy which derived from the feature statistical model for the data. They alternatively estimated the probability density using the Guassian approximation and logistic regression approximation. While, Popuri et al., [7] employs a Dirichlet prior to compute these probabilities, and the probability map is obtained by clustering a feature data sets which included the multimodal images, symmetry, Gabor features, Gaussian, and Laplacian of Gaussian properties.

To improve segmentation accuracy, some algorithms $[8,9]$ exploit a hybrid level set segmentation method driven by region and boundary information simultaneously. In order to get more satisfactory results, some algorithms combined features of the medical images, such as symmetry $[6,7,10]$, texture $[7,11]$, spatial accuracy vector [12], mutual information [13], knowledge-based information [14], and so on. 
According to the derivation of the speed function, these models are mainly separated into edge- and region-based methods. Edge-based contour deformations rely on edge functions of image gradient to identify the tumor boundary. However, this method is adequate in some situations, but it is very sensitive to noisy image, and difficult for the image with blurry edges. For external term of the speed function only depends on the image gradient, pure edge-based methods suffered from the problem of getting trapped into local minima.

More methods extend from a region-based level set method, which can combine statistical information into a tumor segmentation frame. The main difficult point is getting the probabilities of different brain tissues. Therefore, to get satisfactory results, several methods are used for getting probabilities. Since the C-V model is based on the minimum of the intensity difference, it need not compute the probabilities of different tissues. While, the $\mathrm{CV}$ model has some limitations for brain tumor segmentation which is describe in section 1. The present paper describes a level set method with hybrid speed function combined $\mathrm{CV}$ with geodesic length for brain tumor segmentation (GCV).

\section{Data Sets}

To validate the proposed GCV algorithm, the patient MR images used in this study obtained from the Surgical Planning Laboratory (SPL) of the Harvard Medical School \& NSG Brain tumor Database. They consist of ten SPGR T1-weighted volumes of 124 sagittal slices of $256 \times 256$ pixels and $0.9375 \mathrm{~mm} \times 0.9375 \mathrm{~mm} \times 1.5 \mathrm{~mm}$ of voxel size. Ten cases included different types of tumors in different location: case 1-3 are meningioma, 4,6,10 are low-grade glioma, and 5, 7-9 are astrocytoma [16-17].

\section{Geodesic-CV Level Set Method}

In this section, we describe our proposed region and edge-based framework for guiding active contours. Within this framework, segmentations utilize both the global constrains of the intensity difference and local information of the edge gradient. The segmentation formulation is solved as a variational problem by using numerical scheme.

\subsection{Introduction}

The level set method is a numerical algorithm for tracking the evolution of curves. Instead of parametric representations of the curve, the curve is embedded as the zero level set of a higher dimensional function. The function is called the level-set function, $\phi(x(t), t)$. The evolving contour of the level set can be obtained by extracting the zero level-set:

$$
\phi(x(t), t)=0
$$

By the chain rule,

$$
\phi_{t}+\nabla \phi(x(t), t) \cdot x^{\prime}(t)=0
$$

Defining the normal $\vec{N}=\frac{\nabla \phi}{\|\nabla \phi\|}$, and the speed function $x^{\prime}(t) \cdot \vec{N}=F$. The level-set function is then evolved under the control of a differential equation:

$$
\phi_{t}=-F \cdot|\nabla \phi|
$$


Where $F$ describes the speed in the normal direction. $|\nabla \phi|$ is the norm of the gradient of the level set function. During a minimization procedure, the curve is evolved by speed function, which is controlled by certain energy terms.

\subsection{Skull Removal}

Since the skull with high intensity sometimes may affect the segmented results of the tumors, the first progress of segmentation is to remove skull from the brain. After removing the skull, MR images of brain tumor can be divided into four tissue types: white matter (WM), gray matter (GM), ventricular cerebrospinal fluid (CSF), and cortical CSF. For this purpose, a morphology algorithm is performed in the patient's original image and then masked out the skull. A series of the morphological operator was used for delineating the brain tissues without the skull.

\subsection{Segmentation by Combining Region with Edge Information}

Assumed that image is an open and bounded given domain $\Omega$, one dimension or higher dimension. Let be a closed contour $C$ represented as the zero level set of a signed distance function $\phi$, i.e., $[8,9]$. The hybrid variational level set formulation consist of three terms, such as penalizing term, edge term, and region term. Penalizing terms is utilized to avoid that periodically re-initializing the level set function to a signed distance function during the evolution. Region terms represent the intensity difference of inside curve and outside curve. Edge terms are the gradient descent flow that minimizes the geodesic length. The curve evolution the following variational formulation:

$$
E=\lambda_{1} P(\phi)+\lambda_{2} L_{g}(\phi)+\lambda_{3} R(\phi)
$$

where the scalar constants $\lambda_{1}$ and $\lambda_{2}$ are fixed parameters which weighting the relative influence of each of the terms on the evolution of the contour. $P(\phi)$ controls the effect of penalizing the deviation of from a signed distance function, $L_{g}(\phi)$ and $R(\phi)$ are respectively the region energy and edge energy that would drive the motion of the zero level curve of $\phi$.

Region term is derived from the Chan-Vese model, which is based on the intensity difference. It is defined as a global constrain, which is formed as follows:

$$
R(C)=\int_{\text {inside }(C)}\left|I-c_{\text {in }}\right|^{2} d x d y+\int_{\text {ouside }(C)}\left|I-c_{\text {out }}\right|^{2} d x d y
$$

Where $R(C)$ is an energy function, $I(x, y)$ is the intensity of the image at the point $(x$, $y$ ), and the constant parameters $c_{\text {in }}$ and $c_{\text {out }}$ are the average intensity of the image inside and outside the curve $C$ respectively.

The final segmentation, which is only depended on region term, is hardly accurate for the images with heterogeneous intensity even after a long iteration time. This is because that the algorithm is based on the assumption that the image intensity is piecewise constant. Thus, in order to get satisfactory performance for the image with heterogeneous intensity, the edge-terms is needed.

The edge terms is introduced in Geodesic active contour model. It proved that the image segmentation can transfer to find the minimum-length of the zero level set curve of $\phi$ measured in a Riemannian space induced from the image. Its functional to be minimized is stated as follows:

$$
L_{g}(\phi)=\int_{\Omega} g \delta(\phi)|\nabla \phi| d x d y
$$


Here, $\mathrm{g}$ is a decreasing function of the image gradient which computing by an edge indicator function (usually of the form: the reciprocal $\frac{1}{\left(1+\left\|\nabla G_{\delta} * I\right\|\right)^{2}}$ ). The image $I$ is initially convolved with the Gaussian $\operatorname{kernel} G_{\delta}(x, y)=\delta^{1 / 2} e^{-\left|x^{2}+y^{2}\right| / 4 \delta}$, especially for the cases where $I$ is noisy.

In order to keeping stable curve evolution, it is crucial to maintain the evolving level set function as an approximate signed distance function, especially in a neighborhood around the zero level set. Re-initialization has been extensively employed as a numerical remedy for ensuring desirable results in the level set methods. However, it is quite complicated and time-consuming. Meanwhile, it has some undesirable side effect [15]. A signed distance function must satisfy a desirable property of $|\nabla \phi|=1$. Conversely, any function $\phi$ satisfying $|\nabla \phi|=1$ is the signed distance function plus a constant [13].We add a penalizing term to characterize how close a function $\phi$ is to a signed distance function.

$$
P(\phi)=\int_{\Omega} \frac{1}{2}(|\nabla \phi|-1)^{2} d x d y
$$

According to gradient flows,

$$
\frac{\partial \phi}{\partial t}=-\frac{\partial P(\phi)}{\partial \phi}=\operatorname{div}\left(\left(1-\frac{1}{|\nabla \phi|}\right)\right) \nabla \phi
$$

The gradient flow has a factor $(1-1 /|\nabla \phi|)$ which act as diffusion rate. If $|\nabla \phi|>1$, the diffusion rate is positive and the effect of this term is the usual diffusion, i.e., making $\phi$ more even and therefore reduce the gradient $|\nabla \phi|$.If $|\nabla \phi|<1$, the term has effect of reverse diffusion and therefore increase the gradient [15].Thus, the penalty term characterizes how close the level set function $\phi$ is to a signed distance function. Usually, after the energy function comes to a steady state, or approximately to a minimum, the level set function is quite close to a signed distance function.

The level set function $\phi$ that minimizes this functional equation (1) satisfies the EulerLagrange equation. By calculus of variations, the associated curve evolution partial differential equation (PDE) in (1) can be written as:

$$
\frac{\partial \phi}{\partial t}=\lambda_{1}\left[\Delta \phi-\operatorname{div}\left(\frac{\nabla \phi}{|\nabla \phi|}\right)\right]+\lambda_{2} \delta(\phi) \operatorname{div}\left(g \frac{\nabla \phi}{|\nabla \phi|}\right)+\lambda_{3} \delta(\phi)\left[-\left(I-c_{\text {in }}\right)^{2}+\left(I-c_{\text {out }}\right)^{2}\right]
$$

where $\Delta$ is the Laplacian operator, $c_{\text {in }}$ and $c_{\text {out }}$ are, respectively, updated at each iteration by:

$$
\begin{aligned}
c_{\text {in }} & =\frac{\int_{\Omega} I(x, y) H(\phi(x, y)) d x d y}{\int_{\Omega} H(\phi(x, y)) d x d y}, \\
c_{\text {out }} & =\frac{\int_{\Omega} I(x, y)(1-H(\phi(x, y))) d x d y}{\int_{\Omega}(1-H(\phi(x, y))) d x d y} .
\end{aligned}
$$


Where $H_{\varepsilon}$ and $\delta$ are respectively, the approximation of the smoothed Heaviside function and Dirac delta function as:

$$
H_{\varepsilon}(z)=\frac{1}{2}\left(1+\frac{2}{\pi} \arctan \left(\frac{z}{\varepsilon}\right)\right), \delta(z)=\frac{d}{d z} H(z)
$$

Numerical scheme is utilized for solving the partial differential equation. Because of the diffusion term introduced by penalizing energy, the geodesic length of the evolution curve no longer need the upwind scheme as in the traditional level set methods. Instead, all the spatial partial derivatives and are approximated by the central difference, and the temporal partial derivative is approximated by the forward difference.

\subsection{Results}

The proposed model has been performed on the patients of the SPL data sets by Matlab 7 on a computer with Intel Core (TM) i3, 2.13GHz CPU, 4G RAM, and Windows 7 operating system. At each iteration, the removal skull is estimated by the algorithm combing the watershed method with the morphology method. To comfortably place the initial curve, the narrow band threshold level set method is used through the whole image domain. And then the evolution function is computed at each voxel using Eq. (8). Figure 1 describes the major stages of the algorithm. Operator in Oval frame can select not to use.

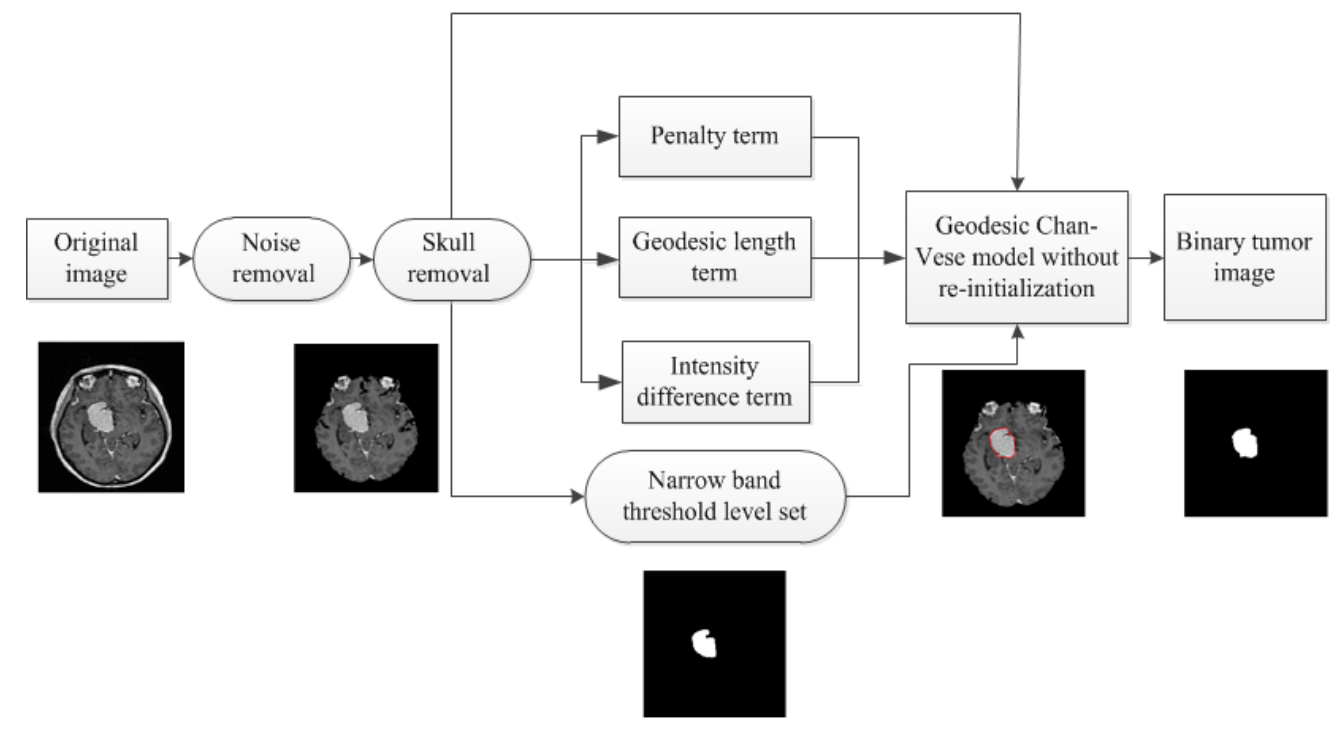

Figure 1. The Major Stages of the Algorithm

We used the similar parameters of the time-step $\Delta t=5$, the grid spacing $\Delta h=1$, the window size of averaging convolution operator $k=15$ for all the experiments in this section. The number of iterations and parameters of the algorithm can be chosen by the user. The couple of controlling parameters of global term and local term $\lambda_{2}, \lambda_{3}$ should be set according to the image intensity property. Generally, we fixed $\lambda_{2}=1$ and dynamically adjusted the value of $\lambda_{3}$. In our experiments, the values of $\lambda_{3}$ are close to 0.3 and need to be dynamically adjusted. It can be found that we were able to get good segmentation results on a wide range of images with these parameters.

Figure 2 describes the Synthetic image with heterogeneous intensity on a background of similar heterogeneous intensity. (a) shows an original image and initial contour. (b) unsuccessful segmented result of region-based model. (c) successful segmented result of 
hybrid model. Intuitively, the hybrid model is more suitable for the intensity in homogeneities.
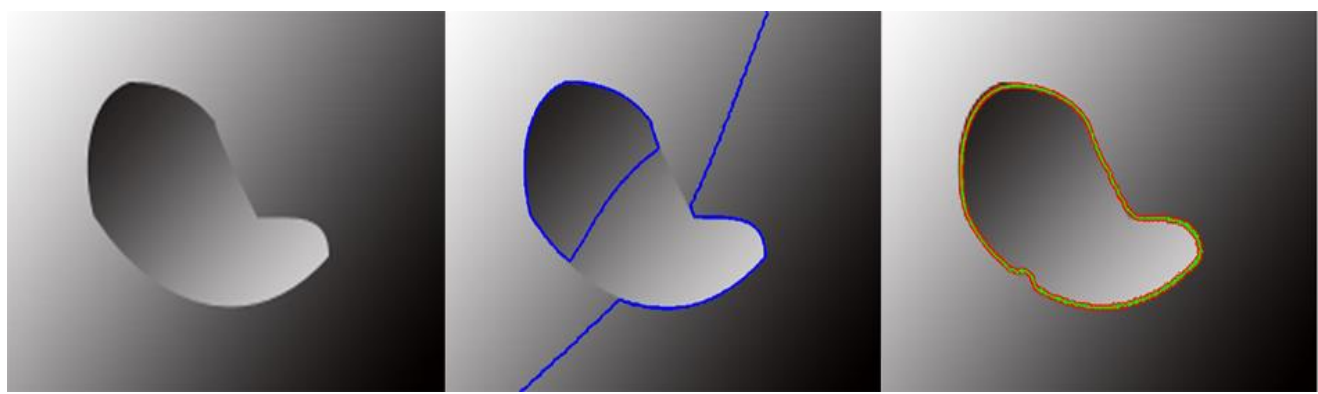

Figure 2. Segmentation Results after Applying the CV Model and the GCV Model on Image with Intensity Heterogeneous

Figure 3 illustrates a comparison of segmentation results of these algorithms on a synthetic image with complex background. The image consists of four objects. The starshape object is desired target boundary among all objects. The intensities of image are segmented into three clusters corresponding to foreground and background, white, gray, and black. From Figure 3, we can find the effect of region-based term and hybrid terms for the segmentation results of $\mathrm{C}-\mathrm{V}$ model and hybrid model. With the similar parameters, the evoluting contour of $\mathrm{C}-\mathrm{V}$ model propagates rapidly towards the black region. The new method can be controlled by the local properties and slow down expanding speed of the zero level set curve which is controlled by the region information.
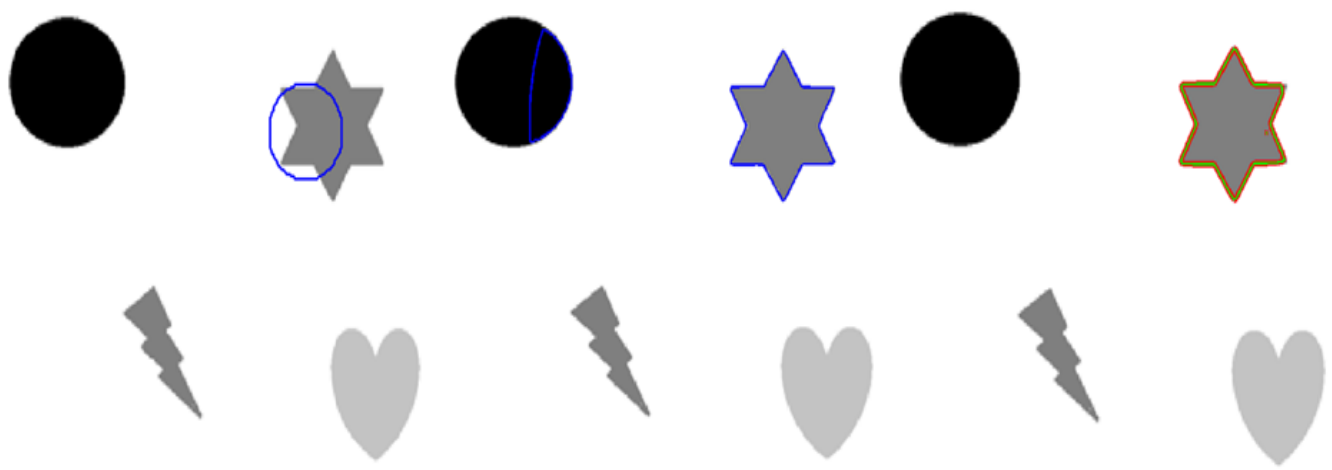

\section{Figure 3. An Example of the Segmentation Result for Synthetic Image with Complex Backgrounds}

Figure 4 shows the contours of the tumor after using geodesic $\mathrm{CV}$ model on some slices of three patients. The data sets containing different kind, size, shape and location of lesions in order to better validate. The segmentation results are described by red contour, and the backgrounds are the skull-stripped images. The segmentation results of the GCV algorithm achieved good performances by visually assessments. 


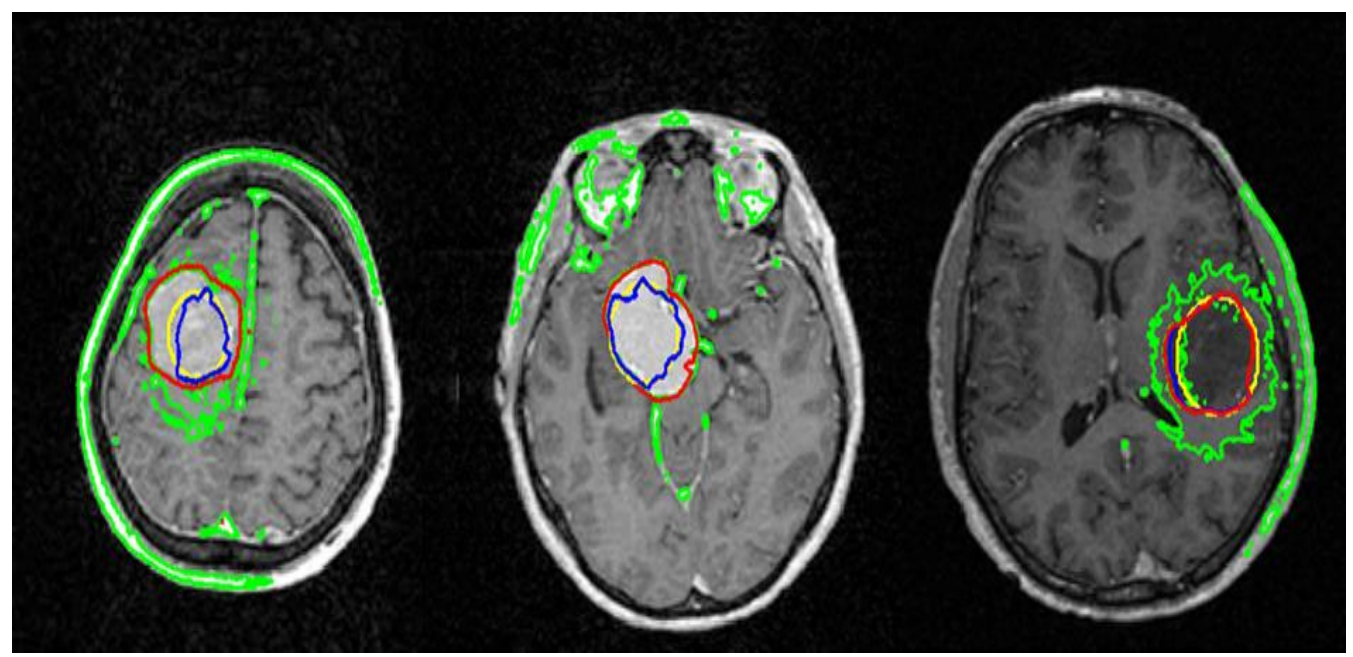

Figure 4. Visually Assessment of the Segmentation Results among the GCV Method, CV Model, and Li Model on Three Slices

To clearly see the significant difference comparing the region-based model, edge-based model with the hybrid model, one slice of each patient is selected to show the segmented results of these three methods (blue, green, and red, respectively). And the initial curve placement is yellow. Visually assessments show the difference among these three methods using for the brain tumor. For instance, adopting the same number of iterations, the classical region term is attracted the contour to the global image domain by the region intensity information. The evoluting curve leaks out the boundary of desired object. The contour driven by the edge terms is fallen in by the local minimum. These are showed on Figure 5.which illustrates the main difference among these methods.

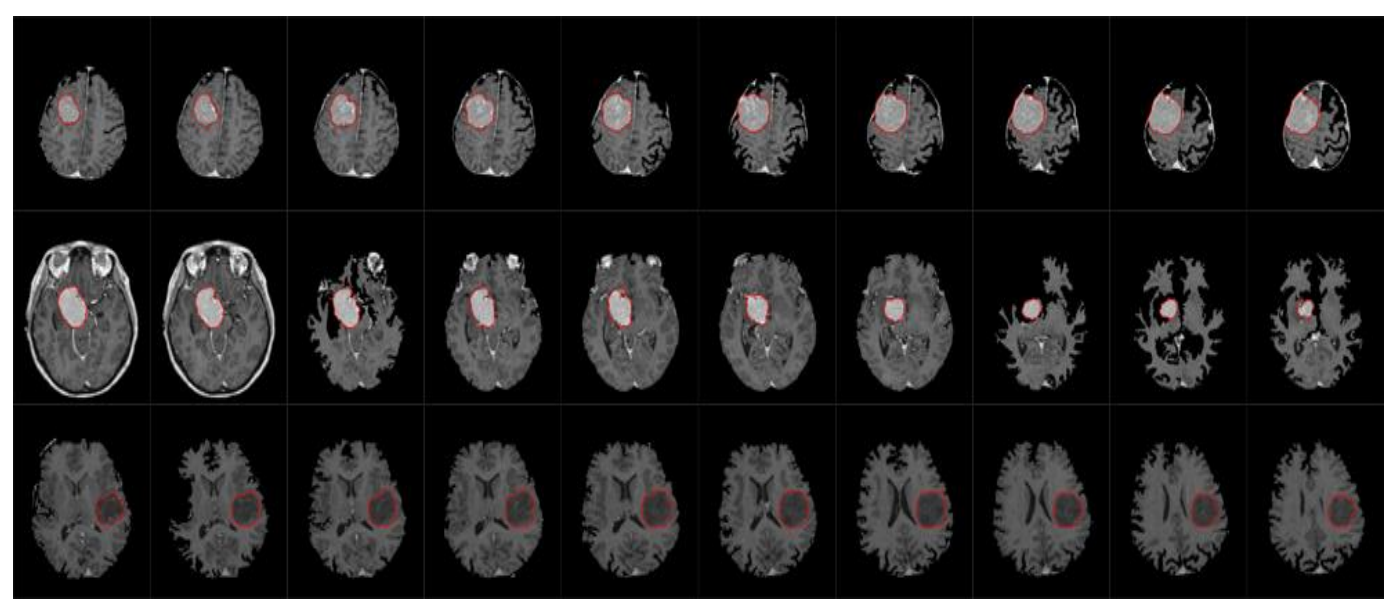

Figure 5. Segmentation Results Obtained after using the Proposed Method on Three Patient Data Sets

\subsection{Quantitative Assessment}

In order to quantitatively assess the registration accuracy, the truths were manually performed in the patient's images by a neuroradiology with expertise on brain tumor images. The performance results of the proposed segmentation frame are assessed by quantitatively comparing with manual contours. 


\section{Table 1. Quantitative assessment for the proposed algorithm by using Jaccard, Dice, False positive ratio, False negative ratio, Sensitivity and Specificity methods}

\begin{tabular}{ccccccc}
\hline Case & Jacard & Dice & False positive ratio & False negative ratio & Sensitivity & Specificity \\
\hline Tumor1 & $0.893 \pm 0.06$ & $0.943 \pm 0.03$ & 0.010 & 0.107 & $0.894 \pm 0.06$ & 0.983 \\
Tumor2 & $0.934 \pm 0.03$ & $0.966 \pm 0.02$ & 0.004 & 0.023 & $0.977 \pm 0.01$ & 0.969 \\
Tumor3 & $0.838 \pm 0.11$ & $0.912 \pm 0.03$ & 0.012 & 0.156 & $0.847 \pm 0.12$ & 0.975 \\
\hline
\end{tabular}

The Jaccard, Dice similarity coefficient, false positive ratio, false negative ratio, sensitivity, and specificity are shown in Table 1 . Let be $T_{p}$ the true positive, $F_{p}$ be the false positive, and $F_{n}$ be the false negative. The Jaccard score is $T_{p} /\left(T_{p}+F_{p}+F_{n}\right)$. The Dice similarity coefficient defined as $D S C=2 T_{p} /\left(2 T_{p}+F_{n}+F_{p}\right)$. The false positive ratio is $F p r=F_{p} /\left(T_{p}+F_{n}\right)$. The false negative ratio is $F n r=F_{n} /\left(T_{p}+F_{n}\right)$. The sensitivity computed as $S e=T_{p} /\left(T_{p}+F_{n}\right)$. The specificity measured by $S p=T_{p} /\left(T_{p}+F_{p}\right)$ Jaccard, Dice, sensitivity and specificity values ranged from 0 to 1 , and were identical to 1 if automatic and manual volumes were equal and the intersection was complete. While the more $F p r$ and $F n r$ close to zero, the more similar between two segmentation images are. Table 1 shows the values of quantitative assessment. For example, the means of Jaccard values were 0.838-0.934 and standard deviation were 0.030.11. Overall, the segmentation results indicate a higher segmentation quality for the tumors without infiltration than with infiltration.

\section{Discussion}

In this paper we have presented a region-based level set method combined with local edge information that allows us to detect the brain tumor in the MR image. Moreover, an optimality penalizing term has been used to avoid the time-consuming re-initialization step of the level set. In order to reduce the affection of skull, a new skull-stripped approach, which incorporates a morphological method, is firstly implemented. After the skull removal, the proposed segmentation method shows good performance for tumor regions as for real tumor cases.

This algorithm is in fact very similar to the Chan-Vese mode in [2] but it solves some of its major limitations using for segmentation of the images with intensity inhomogeneous. Spatial intensity inhomogeneity induced by acquisition process in MR image may cause hollows inside regions, even error segmentation via using the regionbased level set method. A geodesic length term employing the edge gradient function is used in order to attract the curve to the boundary and reduce the dependence of intensity homogeneity. This has been shown in Figure 1 where the contrast products present the new approach has proven to be robust to inhomogeneous intensity distribution.

Regarding the convergence of the evoluting curve, some differences have been observed among Chan-Vese model; Li algorithm and Geodesic Chan-Vese algorithm during the same iteration number (see Figure 2). The evoluting curve of Chan-Vese model is easy to expand to the whole image and pass through the boundary of the desired object. The contour of the edge-based method sometimes converges to the local minimum. While, curve of the proposed algorithm is controlled by both the global region constrains and the local edge properties. Thus, the edge properties increase local constrains into region information and driven the curve converge to a desired boundary for the images with complex objects. 
It is worth noting here, that the penalizing term is used to eliminate the need of the reinitialization. A larger time step can be chosen to speed up the contour evolution, while keep stable evolution of the level set function.

One limitation of this model is shared by Chan-Vese model which want to segment one object from the image with the complex background objects. The algorithm is sensitive to initial placement of curve, which is not too far away from the target object. This is due to that they often lead to a compromise between the global expanding and local shrinkage. To cope with this problem, more initial constraints have to be included in the segment process for instance by incorporating pre-segment in the pre-process. Zhao et al., presented a rough localization algorithm by using Expectation Maximization for the Gaussian Mixed Model. By incorporating the narrowband threshold level set for rough initial localization, the initial placement may closer to the tumor boundary. Of course, this step is not required.

\section{Conclusion}

In this paper, we propose a geodesic Chan-Vese model for brain tumor segmentation, which is based on the curve evolution and level set theory. The energy functional for the proposed model consists of global term of the region intensity difference, local term of the geodesic length and regularization term. By combing the local properties with the region information, the images with heterogeneous intensity and complex background can be efficiently segmented. A penalizing regularization term is introduced into the energy function to avoid the time-consuming re-initialization step. The preliminary results show that the segmented tumor is similar with the manual labeling. The validation and robustness should be further accessed on a larger number of cases. Further improvements of the method could be incorporating more information into the scalar image, such as symmetry of the brain, space correlation of adjacent slices, and the multi- spectral data.

\section{Acknowledgments}

This work was supported by the National Basic Research Program of China (2010CB732505), National Science Foundation Program of China (61272360, 60902103), New Century Excellent Talents in University of Ministry of Education of China (NCET10-0049) and the Plan of Excellent Talent in Beijing (2010D009011000004), project of Ministry of Education of Liaoning (L2012230).

\section{References}

[1] L. A. Vese and T. F. Chan, "A multiphase level set framework for image segmentation using the Mumford and Shah Model", International Journal of Computer Vision, vol. 50, no. 3, (2002), pp. 271293.

[2] M. Droske, M. Bernhard, R. Martin and S. Carlo, "An adaptive level set method for medical image segmentation”, Proceedings of the Information Processing in Medical Imaging (IPMI), Davis, CA, USA, (2001) June 18-22, pp. 416-422.

[3] S. Ho, E. Bullitt and G. Gerig, "Level-set evolution with region competition: automatic 3-d segmentation of brain tumors", Proceedings of the International Conference on Pattern Recognition (ICPR), Quebec, Canada, (2002) August 11-15, pp. 532-535.

[4] M. Prastawa,E. Bullitt, S. Ho and G. Gerig, "A brain tumor segmentation framework based on outlier detection”, Medical Image Analysis. vol. 8, no. 3, (2004), pp. 275-283.

[5] W. Tao, C. Irene and B. Anup, "Fluid vector flow and applications in brain tumor segmentation", IEEE Transactions on Biomedical Engineering, vol. 56, no. 3, (2009), 781-789.

[6] C. Dana and B. Neil, "3D variational brain tumor segmentation using a high dimensional feature set", Proceeding of the IEEE International Conference on Computer Vision (ICCV), Rio de Janeiro, Brasil, (2007) October 14-20, pp. 1-8.

[7] K. Popuri, D. Cobzas and A. Murtha, "3D variational brain tumor segmentation using Dirichlet priors on a clustered feature set”, International Journal of Computer Assisted Radiology and Surgery, vol. 7, no. 4, (2012), pp. 493-506. 
[8] K. Xie, J. Yang, Z. G. Zhang and Y. M. Zhu, "Semi-automated brain tumor and edema segmentation using MRI”, European Journal of Radiology, vol. 56, (2005), pp. 12-19.

[9] W. Cho; J. Park and S. Park, "Level-set segmentation of brain tumors using a new hybrid speed function", Proceeding of the international Conference on Pattern Recognition (ICPR), Istanbul, Turkey, (2010) August 23-26, pp. 1545-1548.

[10] H. Khotanlou and O. Colliot, "3D brain tumor segmentation in MRI using fuzzy classification, symmetry analysis and spatially constrained deformable models", Fuzzy Sets and Systems, vol. 160, (2009), pp. 1457-1473.

[11] C. Daniel, R. Mikael and D. Rachid, "A review of statistical approaches to level set segmentation: Integrating color, texture, motion and shape", International Journal of Computer Vision, vol. 72, no. 2, (2007), pp. 195-215.

[12] J. X. Nie, Z. Xue and T. M. Liu, "Automated brain tumor segmentation using spatial accuracy-weighted hidden Markov Random Field”, Computerized Medical Imaging and Graphics, vol. 33, (2009), pp. 431441.

[13] M. B. Cuadraa, M. D. Craeneb and V. Duay, "Dense deformation field estimation for atlas-based segmentation of pathological MR brain images", Computer Methods and Programs in Biomedicine, vol. 84, (2006), pp. 66-75.

[14] K. E. Emblem, B. Nedregaard and J. K. Hald, "Automatic glioma characterization from dynamic susceptibility contrast imaging: brain tumor Segmentation using knowledge-based fuzzy clustering", Journal of Magnetic Resonance Imaging, vol. 30, (2009), pp. 1-10.

[15] C. Li, C. Xu, C. F. Gui and M. D. Fox, "Level Set Evolution Without Re-initialization: A new variational formulation", Proceedings of the IEEE Computer Society Conference on Computer Vision and Pattern Recognition (CVPR), San Diego, CA, USA, (2005) June 20-26, pp. 430-436.

[16] M. Kaus, S. K. Warfield, A. Nabavi, P. M. Black, F. A. Jolesz and R. Kikinis, "Automated segmentation of MRI brain tumors", Radiology, vol. 218, (2001), pp. 586-591.

[17] K. S. Warfield, M. Kaus, F. A. Jolesz and R. Kikinis, "Adaptive, template moderated, spatially varying statistical classification”, Medical Image Analysis, vol. 4, (2000), pp. 43-55. 
International Journal of Signal Processing, Image Processing and Pattern Recognition Vol.7, No.1 (2014) 